\title{
Diseño incluyente desde el proceso comunicativo usuario-dispositivos para divergencia funcional locomotriz. ${ }^{1}$
}

\section{Inclusive Design from the Communication Process User-Device for Locomotor Functional Divergence. ${ }^{1}$}

\author{
Juan Diego Moreno Arango², Vaslak Rojas Torres ${ }^{3}$ \\ ${ }^{1}$ Instituto Tecnológico Metropolitano de Medellín, Colombia.
}

Artículo recibido en el mes de febrero de 2013; artículo aceptado en el mes de mayo de 2013.

Citación del artículo: Moreno, J. D. \& Rojas, V. (2013). Diseño incluyente desde el proceso comunicativo usuario-dispositivos para divergencia funcional locomotriz. $I+D$ Revista de Investigaciones, 1(1),53-59.

\section{Resumen}

Se realiza una reflexión en torno a la pregunta sobre ¿Cómo mejorar la inclusión de estudiantes con divergencia funcional locomotriz de la ciudad de Medellín?, y a propósito de ello, sobre el tema del análisis comunicacional de la relación sujeto - objeto - contexto, como insumo para una relectura de los dispositivos para personas en situación de discapacidad como agentes facilitadores de la autonomía del usuario y la adaptabilidad a diferentes entornos, y no, como elementos generadores de una dependen- cia funcional. Es decir, que garanticen la completa inclusión de los usuarios en las diferentes dinámicas sociales y en el desarrollo de sus actividades cotidianas. Las personas con divergencia funcional locomotriz requieren del apoyo de dispositivos, planteados como soluciones desde el diseño industrial en este sentido.

Palabras clave: comunicación, divergencia funcional, interface, interacción, percepción de usuario, diseño incluyente.

\footnotetext{
${ }^{1}$ Artículo de reflexión, de enfoque teórico cualitativo, desarrollado a partir del trabajo en el semillero de investigación en diseño y salud, del programa de ingeniería en Diseño Industrial del Instituto Tecnológico Metropolitano de la ciudad de Medellín (Colombia). Calle 73 No 76A - 354 Vía al Volador. PBX: (574) 4405100. La investigación fue financiada por el Instituto Tecnológico Metropolitano.

${ }^{2}$ Comunicador Social-Periodista, Universidad Pontificia Bolivariana de Medellín. Magíster en Salud Pública y Gestión Sanitaria, Universidad de Granada (España). Docente-Investigador, tutor del semillero de investigación: Diseño y salud. Instituto Tecnológico Metropolitano de Medellín (Colombia). Calle 73 No. 76A-354 Vía al Volador. PBX: (574) 4405100. ${ }^{3}$ Diseñador Industrial, Universidad Industrial de Santander. Especialista en Docencia Universitaria, Universidad Industrial de Santander. Docente-Investigador, tutor del semillero de investigación: Diseño y Salud. Instituto Tecnológico Metropolitano de Medellín (Colombia). Calle 73 No 76A-354 Vía al Volador. PBX: (574) 4405100.
} 


\begin{abstract}
In this study, considerations are made regarding the following question, How to improve inclusion of students with locomotor functional divergence in Medellin city?, and about the communicational analysis of the subject-object-context relationship, as a resource for a revision of the devices for people in a locomotor situation of handicap, as facilitator agents of the user's autonomy and their adaptability to different environments, but not as elements that generate a functional dependency, this means, resources that guarantee the full user's inclusion within the different social dynamics and the development of their day-today activities. People with locomotor functional divergence require support by means of several devices, planned as solutions from de industrial design.
\end{abstract}

Keywords: communication, functional divergence, interface, interaction, user perception, inclusive design.

\section{Introducción}

Este documento profundiza en el papel que cumple la comunicación dentro del desarrollo de proyectos de diseño industrial, a partir de la experiencia adquirida en el trabajo realizado en el marco del semillero de investigación en Diseño y Salud del Instituto Tecnológico Metropolitano (ITM) de la ciudad de Medellín; un grupo integrado por estudiantes y docentes que desarrollan proyectos de investigación que convergen en soluciones desde el diseño industrial, para mejorar la calidad de vida de personas con divergencia funcional.

Una de las falencias encontradas, es la exclusión de estas personas de las dinámicas cotidianas en los diferentes contextos en los que participan: familia, estudio, trabajo, ocio entre otros, y las ayudas técnicas o adaptaciones tecnológicas en las cuales se apoyan para realizar sus actividades diarias, en muchas ocasiones potencian esta exclusión, ya que son diseñadas a partir de una concepción funcional y no desde una lectura desde lo comunicacional, donde la relación sujeto - objeto pasa por una dimensión semiológica y sensorial. Las ayudas técnicas se transforman en artefactos culturales que determinan el papel del usuario en un entorno determinado.

En las páginas que siguen, se desglosan los aspectos relacionados con la concepción en torno al dispositivo tecnológico, y cómo ésta se resignifica en el artefacto cultural a través de un sucinto recorrido histórico-conceptual, para luego desarrollar algunas ideas y planteamientos en torno al tema del análisis comunicacional de la relación sujeto - objeto - contexto, como insumo para una relectura de los dispositivos para personas en situación de discapacidad, y finalmente, se presentan algunas conclusiones al respecto.

\section{Del dispositivo tecnológico al artefacto cultural.}

El término dispositivo viene del latín dispositus, y de acuerdo con el diccionario de La Real Academia de la Lengua Española (RAE)

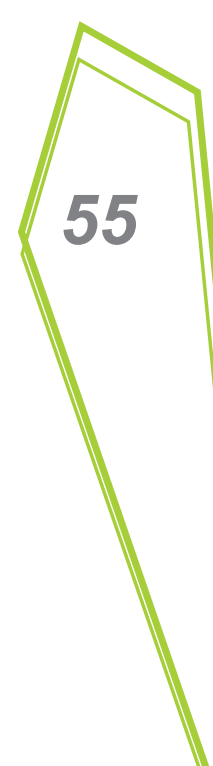


hace referencia a un "mecanismo o artificio dispuesto para producir una acción prevista". Se puede hablar de dispositivo como forma de organización social desde las tecnologías intangibles y dispositivo tecnológico desde una concepción funcionalista del diseño industrial. Esta concepción del dispositivo tecnológico sobresalió en la primera mitad del siglo $\mathrm{XX}$, teniendo como ejemplo el auge de los electrodomésticos para el hogar, el diseño automovilístico, entre otros, que, aunque tuvieron gran efecto en los roles sociales que el hombre y la mujer cumplían dentro y fuera del hogar, no fueron tema de estudio hasta más adelante.

Por otra parte, el término artefactos culturales fue acuñado por los aportes realizados desde la antropología cultural en la segunda mitad del siglo XX, y es un elemento discursivo que hace parte de la cultura y como tal está lleno de significación (Isava, 2009).

Abordar el objeto como elemento discursivo, implica alejarse de una visión del diseño industrial como resultante de la dupla técnico - productiva y estético - formal, y entenderlo como resultante de un ejercicio desde y hacia la cultura, como queda explícito en términos de Dorfles (s.f.):

"no habrá por qué dudar de que un estudio profundo de la relación hombre-objeto a través de las edades, esté en condiciones, mejor que ningún otro, de ofrecernos material histórica y culturalmente revelador acerca de las condiciones socio psicológicas de una determinadacivilización"
Y, en palabras de Pineda (2009), "Los objetos son monumentos, fuentes concretas, significativas y expresivas del estado y las transformaciones de una cultura. Ellos reflejan prácticas, costumbres y gustos de un grupo humano".

De esta manera, para hablar del objeto, se hace indispensable tener en cuenta el contexto en el que fue creado. Bonsieppe (1999), define al diseño como un ejercicio inherente a la cultura, y como tal, parte de un lenguaje, una historia, unos hábitos y costumbres, una ideología y por lo tanto tiene relación directa con el concepto de identidad:

"las cuestiones del diseño no son sólo cuestiones culturales. La identidad cultural es comprensible sólo a través de las categorías del diseño, como objeto para el futuro. Es erróneo abandonarse a un sentimiento de resignación y valorar el diseño con las unidades de medida de la Metrópolis. De este modo se impide ya desde el comienzo la posibilidad de relativizar el concepto de diseño y de reconocer los determinantes históricos".

Las ayudas técnicas para personas con divergencia funcional, como soluciones resultantes de una metodología proyectual en diseño industrial, no son ajenas a esta realidad, y como tal, son artefactos dotados de un discurso y una significación.

\section{Comunicación, interacción y percepción.}

Se entiende la comunicación a partir de la definición que Debray (2001) propone: "comunicar consiste en transportar una 
información dentro del espacio, en el interior de una misma esfera espacio temporal, y transmitir, transportar una información dentro del tiempo, entre esferas espacio temporales distintas".

Desde el Diseño Industrial, hablar de lenguaje objetual es hablar de la comunicación objeto usuario - contexto, la cual está mediada por la percepción del usuario, que se da en gran parte a través de la experiencia de uso. La interfase del objeto es el medio que facilita este proceso comunicacional.

Para Bonsiepe (1999): "Más allá del paradigma comunicativo, está muy difundida la opinión que considera que el objetivo central de la interfase es ayudar al usuario a construirse un modelo mental que reproduce los modelos mentales del programador, quien posee una visión amplia de los detalles operativos del programa" esto en el caso de las computadoras. En el caso de otros objetos, este conocimiento hace referencia al sistema de códigos formales y funcionales utilizados por el diseñador para comunicarse con el usuario; aquí se habla del objeto como mediador entre sujetos y objeto comunicativo.

Pero la comunicación entre diseñador objeto - usuario - contexto, no responde al esquema clásico de comunicación; Norman (2010), propone que más que una comunicación, lo que hay actualmente es una relación unidireccional y que el auge de la interactividad y de los llamados objetos inteligentes no garantizan una comunicación tal como la entendemos desde el punto de vista humano.

\section{Para Norman,}

"los diseñadores pueden pensar que sus productos se comunican pero en el fondo sólo avisan o hacen señales, porque la comunicación se da en una sola dirección. Si queremos cooperar con máquinas autónomas para realizar una tarea conjuntamente, deberemos coordinar nuestras actividades de una manera fluida y agradable".

Como artefacto cultural, un objeto debe comunicar más allá de una identificación "artefactual" o un modo de uso; debe hacerse eco de una historicidad, una identidad cultural o un valor conceptual.

Bal (2006) plantea en su texto Conceptos Viajeros en las humanidades con referencia al texto visual:

"Los conceptos son, y siempre han sido, importantes áreas de debate. Como tales promueven un cierto grado de consenso. Claro que el consenso absoluto no es posible o siquiera deseable, pero si se quiere ir más allá de una mera estrategia defensiva del propio terreno Provisional, tentativo y valorativo - sobre cuál es el mejor significado que se le puede dar a un concepto".

En el caso de las ayudas técnicas para personas con divergencia funcional, éstas son fabricadas con el fin de ser una alternativa de solución o un apoyo para el buen desarrollo de las actividades cotidianas del usuario. Estas ayudas, de alguna forma, se convierten en una extensión

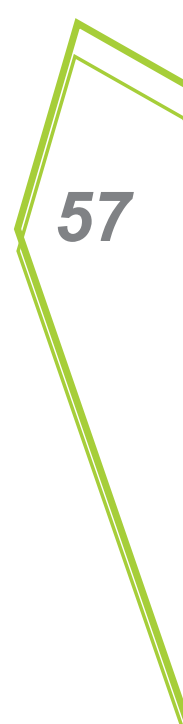


del cuerpo, incluso en algunos casos mimetizándose con éste.

No obstante, la gran mayoría de ayudas técnicas, si bien representan un mejoramiento significativo en la calidad de vida de las personas, no garantizan una inclusión a las diferentes dinámicas del entorno. Ejemplo de ello: la silla de ruedas, que por su altura, no permite que el usuario pueda acercarse a las mesas de cafetería en instituciones educativas, comprar en mostradores, sostener conversaciones con personas que no están a su misma altura o acceder a espacios específicos por sus dimensiones.

Desde el punto de vista del lenguaje objetual, también se puede generar exclusión, ya que el mismo artefacto puede dar la idea de aislamiento por sus características físicas o por sus connotaciones semióticas. Esto se da porque en muchos casos se trata al usuario de estos dispositivos como un espectador del proceso y no como un protagonista del mismo. Es el momento de replantear el rol del usuario en el proceso de proyección, y al igual que plantea Comolli (2007) para el cine "Replantear el papel del espectador".

Algunas metodologías como la $H C D$ (Human Centered Design) de IDEO, proponen un Diseño Centrado en el Usuario, donde él mismo es el protagonista del proceso creativo. Esto no es más que una sinergia entre las herramientas del diseño y las metodologías sociales.

Plantear un diseño incluyente desde la comunicación objetual y no simplemente desde lo funcional, implica hacer una propuesta metodológica que contemple un planteamiento semiótico, o una conceptualización a partir de un sistema de signos que generen un mensaje consciente, o en otros términos, redireccionar el discurso objetual desde su concepción hacia una comunicación incluyente.

\section{Conclusiones}

Entender al objeto como una extensión del ser y desde esta mirada tratar de humanizar el diseño, pasando de una metodología proyectual donde el diseñador plantea unas posibles soluciones a partir de unas necesidades que percibe como agente externo o experto, a incorporar elementos de las ciencias sociales y la comunicación, que permitan ir del sujeto hacia el mismo sujeto, es el reto que se ha impuesto el semillero de investigación en Diseño y Salud del Instituto Tecnológico Metropolitano, en los proyectos de investigación que desarrolla para las personas en situación de discapacidad de la ciudad de Medellín.

Pensar en la inclusión social desde el discurso objetual y no sólo desde las posibilidades técnico funcionales, es resignificar la creación en diseño, generando una relación dialógica entre el sujeto, entorno, diseñador y creación; sólo así se podrá conseguir un verdadero impacto en la forma en que se conciben, se entienden y se usan los dispositivos para personas en situación de discapacidad. 


\section{Referencias}

Bal, M. (2006). Conceptos viajeros en las humanidades. Estudios Visuales. Estética, historia del arte, estudios visuales, 3, 28-77.

Bonsieppe, G. (1999). Del objeto a la interfase. Buenos Aires: Ediciones Infinito.

Comolli, J. L. (2007). "Retrospectiva del Espectador". En Rivera, A., Ver y Aires. Nueva Librería.

Debray, R. (2001). Introducción a la mediología. Barcelona: Editorial Paidós Ibérica.
Dorfles, G. (s.f.) Objeto natural y objeto artificial. Lecturas Morfología Wainhaus. Recuperado de:

http://www.morfologiawainhaus.com/pdf/Dorfl es.pdf

Isava, L. (2009). Breve introducción a los artefactos culturales. Estudios 17:34: 441-454

Norman, D. (2010). El diseño de los objetos del futuro. Barcelona: Editorial Paidós.

Pineda, E. (2009). El objeto de uso como signo. Un recurso para la comprensión de la experencia cotidiana. AdVersus, VI, 14 - 15, 70 99. 